\title{
Nonlinear bulk viscosity and inflation
}

\author{
Roy Maartens* \\ Department of Mathematics, University of Natal, Durban 4041, South Africa \\ and School of Mathematical Studies, Portsmouth University, Portsmouth PO1 2EG, Britain ${ }^{\dagger}$ \\ Vicenç Méndez ${ }^{\ddagger}$ \\ Departament de Fisica, Universitat Autonoma de Barcelona, E-08193 Bellaterra, Spain
}

(November 1996)

\begin{abstract}
We develop a nonlinear generalisation of the causal linear thermodynamics of bulk viscosity, incorporating positivity of the entropy production rate and the effective specific entropy. The theory is applied to viscous fluid inflation (which is necessarily far from equilibrium), and we find thermodynamically consistent inflationary solutions, both exponential and power-law.
\end{abstract}

\section{INTRODUCTION}

Scalar dissipative processes in cosmology may be treated via the relativistic theory of bulk viscosity (see [1], [2] and references cited there). The causal and stable thermodynamics of Israel/ Stewart provides a satisfactory replacement of the unstable and non-causal theories of Eckart and Landau/ Lifshitz. However, it shares with these theories the limitation that it is based on assuming small departures from equilibrium, so that the transport equation is linear in the bulk viscous pressure. While this linear assumption is reasonable for many cosmological and astrophysical situations, there may be processes which involve large deviations from equilibrium. Such deviations are likely to lead to a breakdown of the theory. (This is known to occur for heat flux [3], [4].)

For example, if viscosity-driven inflation occurs (leaving aside for the moment various questions about the hydrodynamical consistency of such models), then this necessarily involves nonlinear bulk viscous pressure [1]. Any application of the linear theories to viscous inflation (see [1] for a review), unavoidably requires an assumption, which is usually not made explicit, that linear thermodynamics remains valid well beyond the regime in which it is derived. The results of such a linear analysis are questionable. The alternative is to develop a nonlinear generalisation of the Israel/ Stewart theory for a more satisfactory model of viscosity-driven inflation. The goal of studying viscositydriven inflation is to find inflationary solutions arising strictly from 'ordinary' matter in a far-from-equilibrium state, without invoking scalar fields. Of course, such models also need to address the question of how the observed density perturbation spectrum can be produced if one eliminates the usual scalr inflaton. This important problem is not addressed here.

We present a phenomenological model of nonlinear bulk viscosity and apply it to viscous inflation. In the absence of a suitable microscopic foundation for a nonlinear theory, we propose a model which at least satisfies reasonable physical constraints, in that it:

1. approaches the Israel/ Stewart theory in the linear regime (i.e. small deviations from equilibrium), thus ensuring causal and stable behaviour in that regime;

2. ensures that the entropy production is non-negative, i.e. the second law of thermodynamics is satisfied;

3. imposes an upper limit on the bulk viscous stress, analogous to existing nonlinear generalisations of the heat flow equation [5].

In Section II we discuss briefly the problem of expanding fluids that are far from equilibrium, especially the case of inflationary expansion. On the basis of the above guiding principles, we then put forward in Section II the mathematically simplest form for a nonlinear transport equation, and consider some of its properties. Hopefully this

\footnotetext{
*maartens@sms.port.ac.uk

${ }^{\dagger}$ permanent address

‡vicenc@ulises.uab.es
} 
simple model can illuminate some of the overall features of a microscopically-based nonlinear theory, which would probably depend heavily on the particular interactions involved. We find in Section III exact solutions in a flat FRW universe, for simple and consistent thermodynamic coefficients and equations of state. Provided the thermodynamic parameters satisfy certain conditions, the model admits thermodynamically consistent inflationary solutions. Although viscous fluid inflation is the only application considered here, the nonlinear theory could in principle be applied to other far-from-equilibrium processes.

\section{TRANSPORT EQUATION FOR BULK VISCOSITY}

The hydrodynamic description which is implicit in fluid dynamics, requires that the mean interaction time $t_{c}$ of fluid particles should be much less than any characteristic macroscopic time-scale. In cosmology, this requires

$$
t_{c} \ll H^{-1},
$$

where $H$ is the Hubble expansion rate. Condition (1) means that the fluid is able to adjust to the cooling caused by expansion, and to establish a state amenable to a hydro-thermodynamical description. In particular, if the fluid is in or close to equilibrium, then (1) should ensure that the fluid has a well-defined local temperature.

For a fluid far from equilibrium, there will not in general be a well-defined temperature. For example, very high and rapidly varying temperature gradients could give rise to large heat flux, without a meaningful temperature at any event. This could happen without violating (1).

If we focus on scalar dissipative effects, in particular ruling out heat flux, then large deviations from equilibrium arise from large bulk viscous stresses, i.e. $|\Pi| \gtrsim p$, where $p$ is the local equilibrium pressure, and the effective non-equilibrium pressure is

$$
p_{\text {eff }}=p+\Pi .
$$

In an expanding fluid, the dissipation due to $\Pi$ leads to a decrease in kinetic energy and therefore in pressure, so that $\Pi \leq 0$.

Qualitatively, bulk viscosity is the macroscopic expression of microscopic 'frictional' effects that arise in mixtures [6], [7]. The bulk viscosity tends to be largest when the contrast between components of the mixture (specifically, the contrast in cooling rates) is greatest. For example:

1. a mixture of ultra-relativistic and non-relativistic particles in an ideal gas in the intermediate regime $m c^{2} \sim k T$;

2. a mixture of photons with long mean free path, and thermalised non-relativistic electrons and protons, whose mean free path is very short (radiative hydrodynamics);

3. a mixture of gauge bosons which have acquired mass after a phase transition in the very early universe, and massless and effectively massless thermalised species.

Bulk viscosity allows us to describe a mixture of different species effectively as a single fluid, provided a hydrodynamic description is reasonable. For example, in radiative hydrodynamics, if the thermalised matter dominates the energy density, then since it obeys (1), we expect that a single-fluid model is reasonable, even though the photon component strongly violates (11). Far from equilibrium, the fluid model itself may break down, in the sense that (11) may not be satisfied by any interactions. We will assume that the deviations from equilibrium are not such as to cause a breakdown in the fluid description. The non-equilibrium conditions could lead to a weakening of (11), i.e. to $t_{c}<H^{-1}$.

In the case of viscous fluid inflation (discussed in more detail in the following section), it is difficult to see how short-range interactions in the fluid could maintain a rate that is greater than the inflationary expansion rate. Fluid particles are being separated at tremendous speed, so that (1) should be strongly violated and a hydrodynamic description should break down. Any consistent fluid model of inflation needs to provide a way around this problem. One possibility could be the existence of long-range interactions, possibly involving a coupling of particle interactions to gravity, whose rate remains higher than the inflation rate. In our subsequent discussion of viscous fluid inflation, we will assume that some such mechanism exists to ensure consistency of the model. (Without such a mechanism being explicit, the model remains phenomenological.) Our task is then to provide a nonlinear transport equation to deal with the large bulk viscous stress involved in inflation.

A covariant approach to the causal thermodynamics of relativistic fluids [8], [9] is based on the hydrodynamic tensors $n^{\alpha}$ (particle number 4 -current), $T_{\alpha \beta}$ (energy-momentum tensor) and $S^{\alpha}$ (entropy 4-current), which are subject respectively to number and energy-momentum conservation 


$$
n_{; \alpha}^{\alpha}=0, \quad T_{; \beta}^{\alpha \beta}=0
$$

and to non-negative entropy production rate (the second law of thermodynamics)

$$
S^{\alpha} ; \alpha \geq 0
$$

The entropy 4 -current is assumed to be algebraically determined by $n^{\alpha}$ and $T_{\alpha \beta}$ alone:

$$
S^{\alpha}=S^{\alpha}\left(n^{\beta}, T^{\mu \nu}\right)
$$

This is a reasonable assumption near equilibrium, and is well-motivated by kinetic theory [8]. Far from equilibrium, (5) could be a drastic assumption, in the sense that the full structure of non-equilibrium states is unlikely to be captured by only the first two moments of the particle distribution. However, for only scalar dissipation, it may be a less restrictive assumption. Furthermore, in homogeneous FRW spacetimes, the assumption that $S^{\alpha}$ does not depend on spatial gradients of the moments will not be restrictive. In any case, without a clear alternative, we can begin by assuming that (5) is also valid for nonlinear scalar deviations from equilibrium.

At each event, one can define a local reference equilibrium state, characterised by number density $n$, energy density $\rho$, pressure $p$, specific entropy $S$ and 4 -velocity $u^{\alpha}$. The arbitrariness in the reference state can be used to match $n$ and $\rho$ to the actual state, in the particle frame, which is characterised by vanishing particle flux, so that

$$
n^{\alpha}=n u^{\alpha}
$$

The energy-momentum tensor for scalar dissipation is then

$$
T_{\alpha \beta}=\rho u_{\alpha} u_{\beta}+(p+\Pi) h_{\alpha \beta},
$$

where $h_{\alpha \beta}=g_{\alpha \beta}+u_{\alpha} u_{\beta}$ is the projection tensor, with $g_{\alpha \beta}$ the metric. In the absence of vector and tensor dissipation, the entropy 4 -current will be of the form

$$
S^{\alpha}=S_{e f f} n^{\alpha}
$$

where $S_{\text {eff }}$ is the effective, non-equilibrium specific entropy.

The conservation equations (3) for (6) and (7) are

$$
\begin{gathered}
\dot{n}+3 H n=0, \\
\dot{\rho}+3 H(\rho+p+\Pi)=0, \\
(\rho+p+\Pi) \dot{u}_{\alpha}+h_{\alpha}{ }^{\beta}(p+\Pi), \beta=0,
\end{gathered}
$$

where $3 H=u_{; \alpha}^{\alpha}$ is the average volume rate of expansion $\left(H=\right.$ Hubble rate in FRW spacetime), and $\dot{u}_{\alpha}=u_{\alpha ; \beta} u^{\beta}$ is the 4-acceleration.

The transport equation for $\Pi$ arises from imposing the second law (4) on (8) - which requires us to specify the form of $S_{\text {eff }}$. In the Israel/ Stewart theory

$$
S_{e f f}=S-\left(\frac{\tau}{2 n T \zeta}\right) \Pi^{2}
$$

where $\zeta(\rho, n)$ is the bulk viscosity and $\tau(\rho, n)$ is the characteristic time for linear relaxational or transient effects. The local equilibrium variables $S$ and $T$ satisfy the Gibbs equation

$$
T d S=(\rho+p) d\left(\frac{1}{n}\right)+\frac{1}{n} d \rho,
$$

which implies, using (9) and (10), that

$$
\dot{S}=-\frac{3 H \Pi}{n T}
$$

It is a feature of the Israel/ Stewart theory that dissipative contributions to the entropy 4-current are quadratic. Any linear contribution is ruled out by the requirement that the entropy density be locally a maximum in equilibrium.

In the Israel/ Stewart theory, the deviations from equilibrium are small, i.e. 


$$
|\Pi| \ll p .
$$

Since $n T \sim p$ and $\tau / \zeta \sim \rho^{-1} \sim p^{-1}$, it follows from (12) and (15) that $S-S_{\text {eff }} \ll 1$, so that (15) prevents the effective specific entropy from becoming negative. We will not impose the constraint (15) on $|\Pi|$. However, for simplicity, we will retain the quadratic form (12), i.e. we will not add cubic and higher contributions, since there is no clear indication as to what such contributions would be - and since they would introduce further, unknown coupling coefficients. Since we require non-negative $S_{\text {eff }}$, a consequence of adopting (12) in the nonlinear case is that the bulk stress is bounded:

$$
S_{e f f} \geq 0 \quad \Leftrightarrow \quad|\Pi| \leq|\Pi|_{\max }=\sqrt{\frac{2 n T S \zeta}{\tau}} .
$$

Apart from this new feature, the main difference between the nonlinear theory presented here, and the linear Israel/ Stewart theory, reduces to the different ways in which the second law is imposed.

By (9), (10) and (13), we find that (8) and (12) lead to

$$
\begin{aligned}
S_{; \alpha}^{\alpha} & =-\frac{\Pi}{T} \mathcal{X} \quad \text { where } \\
\mathcal{X} & =3 H+\frac{\tau}{\zeta} \dot{\Pi}+\frac{\tau}{2 \zeta} \Pi\left(3 H+\frac{\dot{\tau}}{\tau}-\frac{\dot{\zeta}}{\zeta}-\frac{\dot{T}}{T}\right) .
\end{aligned}
$$

In the Israel/ Stewart theory, the second law (田) is satisfied by assuming a linear relation between the thermodynamic 'force' $\mathcal{X}$ and the thermodynamic 'flux' $\Pi$, i.e.

$$
\Pi=-\zeta \mathcal{X} \quad(\text { Israel/ Stewart }) .
$$

(The linearity may be justified via near-equilibrium kinetic theory [8].) Formally, it follows from (18) that $|\Pi|$ is unbounded in response to growth in $\mathcal{X}$. The near-equilibrium condition (15) has to be taken as an additional, extraneous constraint on the transport equation (18). This is a sign of the limitations of the theory arising from its linearity. Beyond near-equilibrium conditions, the linear transport equation (18) cannot be expected to give reasonable predictions.

The nonlinear generalisation of (18) which we propose must be consistent with the upper bound (16) on $|\Pi|$, so that while $|\Pi| / p$ is not restricted to be small, it does reach a limit as the thermodynamic force is increased without bound. This seems to be physically reasonable, and is in line with existing phenomenological generalisations of the heat transport equation [5]. The simplest generalisation of (18) that we could find with this property, and the other properties listed in the introduction, is

$$
\Pi=-\frac{\zeta \mathcal{X}}{1+\tau_{*} \mathcal{X}} \quad(\text { nonlinear })
$$

where $\tau_{*} \geq 0$ is a characteristic time-scale for nonlinear effects. Nonlinear effects are significant for $\mathcal{X} \gtrsim \tau_{*}^{-1}$.

For small thermodynamic force $\mathcal{X}$, equivalently small |ח|, (19) tends to the form (18), and the linear Israel/ Stewart theory is recovered. For $|\mathcal{X}| \rightarrow \infty,-\Pi$ tends to the upper bound $\zeta / \tau_{*}$. It follows from (17) and (19) that

$$
S_{; \alpha}^{\alpha}=n \dot{S}_{e f f}=\frac{\Pi^{2}}{T \zeta}\left[1+\frac{\tau_{*}}{\zeta} \Pi\right]^{-1} .
$$

This means that the second law holds identically by virtue of the upper bound on the bulk stress:

$$
S_{; \alpha}^{\alpha} \geq 0 \quad \Leftrightarrow \quad-\Pi \leq \frac{\zeta}{\tau_{*}}
$$

As $-\Pi$ approaches $\zeta / \tau_{*}$, the entropy production rate $(20)$ tends to infinity, providing a barrier against values $-\Pi>$ $\zeta / \tau_{*}$. Note also that (20) implies that the rate of growth of effective specific entropy is greater in the nonlinear than in the linear theory. The condition (16) implies by (21) that

$$
\tau_{*} \geq \sqrt{\frac{\zeta \tau}{2 n T S}} .
$$

By (17), the detailed form of the transport equation (19) is 


$$
\begin{aligned}
& \tau \dot{\Pi}\left(1+\frac{\tau_{*}}{\zeta} \Pi\right)+\Pi\left(1+3 \tau_{*} H\right) \\
& =-3 \zeta H-\frac{1}{2} \tau \Pi\left[3 H+\frac{\dot{\tau}}{\tau}-\frac{\dot{\zeta}}{\zeta}-\frac{\dot{T}}{T}\right]\left(1+\frac{\tau_{*}}{\zeta} \Pi\right) .
\end{aligned}
$$

The Israel/ Stewart theory, in its full (non-truncated) form [1], [2], is the limiting case $\tau_{*}=0$, when the terms in round brackets in (23) all reduce to 1.

The thermodynamic coefficients $\zeta$ and $\tau$ are known from the linear theory, based on kinetic theory arguments [8]. For the nonlinear coefficient $\tau_{*}$ we do not have a corresponding microscopic derivation. However the phenomenological theory presented here imposes on $\tau_{*}$ the limit (22), which is determined by the linear coefficients and local equilibrium variables.

\section{THERMODYNAMICALLY CONSISTENT INFLATION}

We specialise now to a flat FRW universe

$$
d s^{2}=-d t^{2}+a(t)^{2}\left(d x^{2}+d y^{2}+d z^{2}\right),
$$

where $H=\dot{a} / a$. The transport equation (23) implies, with the Einstein field equations, an evolution equation for $H$, once we specify the thermodynamic coefficients and equations of state. The field equations are

$$
\begin{aligned}
H^{2} & =\frac{1}{3} \rho, \\
\dot{H}+H^{2} & =-\frac{1}{6}(\rho+3 p+3 \Pi) .
\end{aligned}
$$

For the equilibrium pressure we impose the linear barotropic equation of state

$$
p=(\gamma-1) \rho,
$$

where $\gamma$ is a constant, $1 \leq \gamma \leq 2$. If $T$ is also barotropic, $T=T(\rho)$, then the integrability condition $\partial^{2} S / \partial \rho \partial n=$ $\partial^{2} S / \partial n \partial \rho$ of the Gibbs equation (13) leads to [10

$$
T \propto \rho^{(\gamma-1) / \gamma} .
$$

We note that the ideal gas law $p=n T$ is incompatible with barotropic temperature and the linear barotropic equation of state (26), unless $\Pi=0$ [11]. This follows from (27) - which is a consequence of (26) and $T=T(\rho)-$ and the conservation equations (9) and (10).

The linear relaxation time is related to the bulk viscosity by 10

$$
\tau=\frac{\zeta}{v^{2}(\rho+p)}=\frac{\zeta}{v^{2} \gamma \rho}
$$

where $v$ is the dissipative contribution to the speed of sound $V$, so that $V^{2}=c_{s}^{2}+v^{2}$, with $c_{s}$ the adiabatic contribution. By causality, $V \leq 1$, so that

$$
v^{2} \leq 1-c_{s}^{2}=2-\gamma,
$$

where we used (26). We assume that $v$ is constant, like $c_{s}$ is when (26) holds. The bulk viscosity itself is often taken to be of the simplified form $\zeta \propto \rho^{q / 2}$ ( $q$ constant), which should not be unreasonable in FRW spacetime with (26). By (24) we have

$$
\zeta=\alpha H^{q},
$$

where $\alpha(\geq 0)$ is constant. Finally, we need to specify the nonlinear characteristic time $\tau_{*}$. The simplest possibility appears to be

$$
\tau_{*}=k^{2} \tau,
$$

where $k$ is constant, and $k=0$ is the linear (Israel/ Stewart) case. This ansatz is subject to the condition (22), which does not take a simple form in general. 
From (24) - 26) we get

$$
\Pi=-2 \dot{H}-3 \gamma H^{2} .
$$

Together with (27) - (31), this brings (23) to the form

$$
\begin{aligned}
& {\left[1-\frac{k^{2}}{v^{2}}-\left(\frac{2 k^{2}}{3 \gamma v^{2}}\right) \frac{\dot{H}}{H^{2}}\right]\left\{\ddot{H}+3 H \dot{H}+\left(\frac{1-2 \gamma}{\gamma}\right) \frac{\dot{H}^{2}}{H}+\frac{9}{4} \gamma H^{3}\right\}} \\
& +\frac{3 \gamma v^{2}}{2 \alpha}\left[1+\left(\frac{\alpha k^{2}}{\gamma v^{2}}\right) H^{q-1}\right] H^{2-q}\left(2 \dot{H}+3 \gamma H^{2}\right)-\frac{9}{2} \gamma v^{2} H^{3}=0 .
\end{aligned}
$$

This is the fundamental dynamical equation for nonlinear bulk viscosity in a flat universe. Once (33) is solved for $H$, $\rho$ is determined by (24) and $\Pi$ by (32). When $k=0$, the terms in square brackets all reduce to 1 , and we recover the dynamical equation for the (non-truncated) Israel/ Stewart theory [1].]

Viscous inflation has been discussed in a number of papers (see $[1]$ for a review), both as a phenomenological model of quantum particle and string creation effects, and as a fluid alternative to scalar-field inflation. Particle production is formally equivalent to an effective bulk viscosity [2], but we will confine ourselves here to discussing fluid models, in which the bulk viscous stress $|\Pi|$ has become large enough to make the effective pressure (2) negative and initiate inflation, and where net particle creation and decay occur only during reheating, at the end of inflation. (Causal linear thermodynamics, incorporating net particle production, may be used as a phenomenological model of reheating [12].) As discussed in the previous section, we assume that long-range interactions or some other microscopic mechanism are present to ensure that a hydrodynamical description remains reasonable during inflation.

The condition for inflation $\ddot{a}>0$ implies by (25) that

$$
-\Pi>p+\frac{1}{3} \rho .
$$

It is clear from (34) that viscous fluid inflation is not close to equilibrium, i.e. it strongly violates the condition (15), and we need to use the generalised nonlinear equation (33). We consider first whether (33) admits exponential inflation, and if so, whether the solutions conform to the thermodynamic conditions discussed above.

Setting $H=H_{0}$, (33) gives

$$
H_{0}^{1-q}=\frac{\alpha}{2 \gamma v^{4}}\left(2 v^{2}-1\right)\left(v^{2}-k^{2}\right) .
$$

By (32) and (24), $\Pi=-3 \gamma H_{0}^{2}=-\gamma \rho$. Then the condition (21) implies, using (28) and (30), a limit on $k$ :

$$
k \leq v .
$$

In order to check the condition (22), we evaluate the specific entropy. Integrating (14) with $T=T_{0}=$ constant:

$$
S=S_{0}+\left(\frac{3 \gamma H_{0}^{2}}{n_{0} T_{0}}\right) e^{3 H_{0} t},
$$

where $n=n_{0} a^{-3}$. Then (12) leads to

$$
S_{\text {eff }}=S_{0}+\left(\frac{3 \gamma H_{0}^{2}}{2 n_{0} T_{0} v^{2}}\right)\left(2 v^{2}-1\right) e^{3 H_{0} t} .
$$

Thus we require $v^{2} \geq \frac{1}{2}$. It follows from (35), (36) and (29) that exponential inflation is possible provided

$$
k<v, \quad \frac{1}{2}<v^{2} \leq 2-\gamma,
$$

and then the entropy generation and effective specific entropy are positive. Thus the solutions are thermodynamically consistent. Note that $\gamma<\frac{3}{2}$. Exponential growth of $S$ is also found in the linear theory [1] - but the advantage here is

\footnotetext{
${ }^{1}$ Note that [1] effectively assumes $v^{2}=1 / \gamma$, and takes the exponent in (27) to be a free parameter $r$; one must set $r=(\gamma-1) / \gamma$ in [1].
} 
that the theory is nonlinear, and we consider the effective specific entropy, which is more suitable far from equilibrium. The apparent closeness of the linear and nonlinear results is an artefact of the constancy of $H$ and thereby of $\rho, T$, $\Pi$. As shown in [1], it is possible to generate a large amount of entropy via the exponential form (38).

Following the qualitative analysis techniques for the Israel/ Stewart solutions [11], [13], we find that the stability properties of the solution (35) depend on the parameter

$$
\omega=\frac{\left(2 v^{4}+v^{2}-2 k^{2}\right)^{2}}{4 \gamma\left(v^{2}-k^{2}\right)^{2}\left(2 v^{2}-1\right)}>0
$$

Then the fixed point $\left(0, H_{0}\right)$ in the phase plane $\{(\dot{H}, H)\}$ has the following properties:

for $1-\omega \leq q<1$, it is an asymptotically stable node;

for $q>1$, it is a saddle point;

for $q<1-\omega$, it is an asymptotically stable focus.

Thus the solution is an attractor for $q<1$.

When $q=1$, exponential inflation can occur with $H_{0}$ arbitrary, provided that the bulk viscous parameter satisfies

$$
\alpha=\frac{2 \gamma v^{4}}{\left(2 v^{2}-1\right)\left(v^{2}-k^{2}\right)}
$$

as follows from (35). Of more interest is the existence when $q=1$ of power-law solutions $a=a_{0} t^{N}$, which are inflationary for $N>1$. By (33), these solutions exist if

$$
\begin{aligned}
& 27 \gamma^{3}\left[\alpha\left(1-2 v^{2}\right)\left(v^{2}-k^{2}\right)+2 \gamma v^{4}\right] N^{3} \\
& +18 \gamma^{2}\left[3 \alpha k^{2}-2 \gamma v^{4}-2 \alpha v^{2}\left(1+k^{2}\right)\right] N^{2} \\
& +12 \alpha \gamma\left(v^{2}-3 k^{2}\right) N+8 \alpha k^{2}=0 .
\end{aligned}
$$

Clearly there are solutions of this cubic for a wide range of thermodynamic parameters. However, we are concerned to identify the thermodynamically consistent solutions, for which the conditions are stringent.

By (32), $\Pi=-N(3 \gamma N-2) t^{-2}$, and together with (24), (27), (28) and (31), this shows that the condition (21) for positive entropy generation is

$$
k \leq\left(\frac{3 \gamma N}{3 \gamma N-2}\right)^{1 / 2} v
$$

Integrating (14) we get

$$
S=S_{0}+\left[\frac{\gamma\left(3 N^{2}\right)^{1 / \gamma}}{n_{0} T_{0} a_{0}^{3}}\right] t^{(3 \gamma N-2) / \gamma}
$$

and then (12) gives

$$
S_{e f f}=S_{0}+\left[\frac{\left(3 N^{2}\right)^{(1-\gamma) / \gamma}}{6 \gamma v^{2} n_{0} T_{0} a_{0}^{3}}\right]\left\{9 \gamma^{2}\left(2 v^{2}-1\right) N^{2}+12 \gamma N-4\right\} t^{(3 \gamma N-2) / \gamma}
$$

The effective specific entropy is positive if the $N$-quadratic in braces is positive. This requires that

$$
v \leq \frac{1}{\sqrt{2}}
$$

and it places an upper limit on $N$, so that

$$
\frac{2}{3 \gamma}<N \leq \frac{2}{3 \gamma(1-\sqrt{2} v)}
$$

(The lower limit ensures that $\Pi<0$.)

Thus the constraints (43), (45) and (46) must be satisfied for any solution of (42) to be thermodynamically consistent. We present a simple example to show that consistent inflationary solutions do exist:

$$
\gamma=\frac{4}{3}, \quad v^{2}=\frac{1}{2}=k^{2}, \quad \alpha=\frac{67}{4}, \quad N=2 .
$$


In conclusion, we have argued for a nonlinear generalisation of causal linear thermodynamics in order to describe viscous inflation (without particle production). A simple phenomenological theory, supplemented by a simple model for the thermodynamic coefficients and equations of state, leads to severe restrictions if thermodynamical consistency is imposed. However, consistent solutions do exist, both exponential and power-law. These solutions respect the second law of thermodynamics and have positive effective specific entropy. Of course, the phenomenological theory lacks a microscopic foundation, and it is necessary to assume a priori that long-range interactions or some other mechanism are present in order to maintain the viability of a thermo-hydrodynamical description during inflation. If the theory presented here were applied to non-inflationary processes that are far from equilibrium (e.g. in astrophysics), then this limitation would not apply.

\section{ACKNOWLEDGMENTS}

Thanks to David Jou for useful discussions. RM thanks the Department of Physics at Barcelona (especially Diego Pavón) and the Department of Mathematics at Natal (especially Sunil Maharaj) for warm hospitality. RM was supported by research grants from Natal and Portsmouth Universities, and VM by the Spanish Ministry of Education under grant PB94-0718.

[1] R. Maartens, Class. Quantum Grav. 12, 1455 (1995).

[2] W. Zimdahl, Phys. Rev. D 53, 5483 (1996).

[3] W.A. Hiscock and L. Lindblom, Phys. Lett. A 131, 509 (1988).

[4] M. Criado-Sancho and J.E. Llebot, Phys. Lett. A 177, 323 (1993).

[5] D. Jou, J. Casas-Vazquez and G. Lebon, Extended Irreversible Thermodynamics (Berlin: Springer, 1996).

[6] N. Udey and W. Israel, Mon. Not. R. Astr. Soc. 199, 1137 (1982).

[7] W. Zimdahl, Mon. Not. R. Astr. Soc. 280, 1239 (1996).

[8] W. Israel and J. Stewart, Ann. Phys. 118, 341 (1979).

[9] W.A. Hiscock and L. Lindblom, Ann. Phys. 151, 466 (1983).

[10] R. Maartens, in Proceedings of Hanno Rund Workshop, to appear; astro-ph/9609119 (1996).

[11] V. Méndez and J. Triginer, J. Math. Phys. 37, 2906 (1996).

[12] W. Zimdahl, D. Pavon and R. Maartens, to appear Phys. Rev. D; astro-ph/9611147 (1996).

[13] A.A. Coley, R. Van den Hoogen and R. Maartens, Phys. Rev. D 54, 1393 (1996). 